NBER WORKING PAPER SERIES

\title{
TESTING FOR CHANGES IN THE SES-MORTALITY GRADIENT WHEN THE DISTRIBUTION OF EDUCATION CHANGES TOO
}

\author{
Thomas Goldring \\ Fabian Lange \\ Seth Richards-Shubik \\ Working Paper 20993 \\ http://www.nber.org/papers/w20993
NATIONAL BUREAU OF ECONOMIC RESEARCH
1050 Massachusetts Avenue
Cambridge, MA 02138
February 2015

Thomas Goldring was supported in part by Carnegie Mellon University's Program in Interdisciplinary Education Research (PIER) funded by grant number R305B090023 from the U.S. Department of Education. This paper uses data obtained from the public-use file of the National Longitudinal Mortality Study. The file is provided to persons interested in research by the U.S. Census Bureau. The views expressed in this paper are those of the authors and do not necessarily reflect the views of the National Longitudinal Mortality Study, the Bureau of the Census, or the project sponsors: the National Heart, Lung, and Blood Institute, the National Cancer Institute, the National Institute on Aging, and the National Center for Health Statistics. The views expressed herein are those of the authors and do not necessarily reflect the views of the National Bureau of Economic Research.

NBER working papers are circulated for discussion and comment purposes. They have not been peerreviewed or been subject to the review by the NBER Board of Directors that accompanies official NBER publications.

(C) 2015 by Thomas Goldring, Fabian Lange, and Seth Richards-Shubik. All rights reserved. Short sections of text, not to exceed two paragraphs, may be quoted without explicit permission provided that full credit, including $(\mathrm{C}$ notice, is given to the source. 
Testing for Changes in the SES-Mortality Gradient When the Distribution of Education Changes

Too

Thomas Goldring, Fabian Lange, and Seth Richards-Shubik

NBER Working Paper No. 20993

February 2015

JEL No. I14,J11

\begin{abstract}
We develop a flexible test for changes in the SES-mortality gradient over time that directly accounts for changes in the distribution of education, the most commonly used marker of SES. We implement the test for the period between 1984 and 2006 using microdata from the Census, CPS, and NHIS linked to death records. Using our flexible test, we find that the evidence for a change in the education-mortality gradient is not as strong and universal as previous research has suggested. Our results indicate that the gradient increased for females during this time period, but we cannot rule out that the gradient among males has not changed. Informally, the results suggest that the changes for females are mainly driven by the bottom of the education distribution.
\end{abstract}

Thomas Goldring H. John Heinz III College

School of Public Policy and Management

Carnegie Mellon University

5000 Forbes Avenue

Pittsburgh, PA 15213

tlpg@cmu.edu

Fabian Lange

McGill University

Department of Economics

855 Sherbrooke Street West

Montreal, Quebec, H3A 2T7

fabolange@gmail.com
Seth Richards-Shubik

H.John Heinz III College

School of Public Policy and Management

Carnegie Mellon University

5000 Forbes Avenue

Pittsburgh, PA 15213

and NBER

sethrs@andrew.cmu.edu 


\section{Introduction}

Persistent differences in health and longevity across socioeconomic status (SES) are wellestablished, and a growing literature claims that these differences have increased over recent decades. ${ }^{1}$ Some authors further suggest that in the U.S. longevity among some low SES groups, as measured by education, declined not just relative to high SES groups, but also in absolute terms. Olshansky et al. (2012, p. 1808), for instance, claim that "along the educational and socioeconomic status gradient, those at the top are gaining modest amounts of longevity, but whites at the bottom are losing ground at a faster pace - that is, they are either experiencing a decline in life expectancy or a slower rate of increase relative to those at the top." 2 The evidence for these claims stems largely from comparing mortality rates across categories of educational attainment over time.

However, at the same time that mortality rates conditional on education changed, the distribution of educational attainment in the U.S. has also changed dramatically. For example, among women aged between 25 and 84, the share with less than a high school diploma declined from $25.8 \%$ to $14.0 \%$ between 1984 and 2006 (Table 1). These large changes in the distribution of education imply that the absolute level of education cannot be a stable marker of SES over time. This raises the question of what it means to measure changes in the SESmortality gradient by comparing mortality rates across education categories. For example, over the last 35 years, longevity among those with "less than a high school diploma" declined relative to the more educated, suggesting that the SES gradient of mortality widened. However, having "less than a high school diploma" today also means belonging to a smaller and potentially more disadvantaged group. So does the relative decline in longevity for this group truly represent a widening in the SES gradient of mortality? Fundamentally, the question becomes whether it is possible to infer how the SES gradient in mortality changes over time using mortality rates conditional on education given that the education distribution itself has changed so dramatically. ${ }^{3}$

\footnotetext{
${ }^{1}$ The literature originates with Kitagawa and Hauser (1973). For a comprehensive recent review, see Hummer and Lariscy (2011).

${ }^{2}$ Most of the literature uses educational attainment as the principal indicator of SES in order to examine socioeconomic differentials in adult mortality (see, for example, Hummer and Lariscy, 2011; Olshansky et al., 2012). There are two main reasons for this. First, contrary to occupation and work-related measures of SES, education is available as a measure of SES for the retired, the unemployed, and those out of the labor force. Second, education is fixed throughout adulthood and thus less vulnerable to issues of reverse causality when examining its relation with health among adults (Preston and Taubman, 1994).

${ }^{3}$ Dowd and Hamoudi (2014) discuss this issue in a critique of the literature, and Olshansky (2013) acknowledges the problem. However we are not aware of any work that has proposed how to account for
} 
Table 1: Female mortality rates by educational attainment and time period

\begin{tabular}{lcccccc}
\hline & \multicolumn{2}{c}{$1984-89$} & & \multicolumn{2}{c}{ 2002-06 } \\
\cline { 2 - 3 } \cline { 5 - 6 } Educational & Population & Mortality & & Population & Mortality \\
Attainment & Share & Rate & & Share & Rate \\
\hline Less than grade 12 & 0.258 & 0.060 & & 0.140 & 0.064 \\
Grade 12 & 0.426 & 0.050 & & 0.402 & 0.045 \\
More than grade 12 & 0.317 & 0.044 & & 0.458 & 0.036 \\
\hline
\end{tabular}

Notes: These figures are calculated from the National Longitudinal Mortality Study and the National Health Interview Survey - Mortality Linked Files (see Section 3 for details). The mortality rate is the average probability of death within the 5-year time period at the top of the column (the period 1984-89 runs from April 1984 to March 1989, whereas 2002-06 runs from January through December). All statistics are standardized by age, race, and region to the distribution in the 2000 NHIS.

In this paper, we develop a new method to investigate changes in the relationship between mortality and SES, while accounting for changes in the distribution of education over time. The data directly inform us about (i) the share of the population in each education group, and (ii) mortality conditional on educational attainment. We propose a nonparametric test to assess whether the observed education shares and mortality rates at two points in time could be consistent with the same underlying relationship between SES and mortality. We then apply this test to data from the U.S. covering the period from 1984 to 2006. As detailed below, we confirm the standard conclusion that the gradient has increased for females. However we fail to find evidence that the gradient has increased for males. This contrasts with a number of studies, including our own, that have found increases in the gradient for males (under different-typically parametric - specifications).

To demonstrate the concurrent changes in education and mortality, Table 1 summarizes education shares and mortality rates for females aged between 25 and 84 in 1984-89 and 2002-06. ${ }^{4}$ In just two decades, the fraction who did not complete grade 12 decreased sharply from 0.258 in 1984-89 to 0.140 in 2002-06. Conversely, the fraction with at least some college education increased from 0.317 to 0.458 . For mortality rates, Table 1 displays two important patterns. First, in both time periods mortality rates fall with educational attainment. Second, mortality rates conditional on education decline over time for those who these shifts in the distribution of education in a systematic fashion.

${ }^{4}$ Details on the data used to construct this table can be found in Section 3 below. 
completed 12 years of education as well as those who completed more than 12 years of education. The decline is more pronounced among the latter. At the same time, mortality rates increased among those with less than 12 years of education. Together, these changes imply that between 1984 and 2006 the mortality gradient in absolute levels of education increased.

But has the underlying SES-gradient in mortality also increased? Having less than a high school diploma in 2002-06 implies being in the bottom $14.0 \%$ of the education distribution, whereas in 1984-89 it meant being in the bottom 25.8\%. It is likely that in 2002-06, as compared to 1984-89, those with less than a high school diploma are less favorably selected from the population in terms of their social status and economic resources. Thus the differences in mortality rates between this group and the rest of the population might widen not because the underlying SES-mortality gradient increased, but rather because this group is increasingly drawn from lower in the SES distribution. This leads to an empirical problem in determining from observed changes in the education-mortality relationship whether the underlying SES-mortality gradient has truly increased or whether the observed changes are entirely due to the change in the distribution of education. Our nonparametric test is specifically intended to address this issue.

The testing procedure relies on two assumptions that are implicit in much of the literature: that individuals can be ranked by their SES; and that education is a primary marker of SES, in the sense that those with more education are assumed to have greater SES. These assumptions allow us to determine the range in the SES distribution to which any individual belongs. For example, from the data in Table 1, a female with less than 12 years of education in 1984-89 is known to be part of the bottom 25.8\% of the SES distribution. By contrast, a female with 12 years of education ranks above the bottom $25.8 \%$ and below the top $31.7 \%$. A final assumption we require is that mortality declines (weakly) with rank in the SES distribution. Given these assumptions, we derive a non-parametric test of the NullHypothesis that the underlying relationship between SES and mortality is unchanged.

Our test works as follows. In each time period, the education distribution implies a partition of the SES distribution, and we can estimate the mortality rate in each element of this partition. Under the null hypothesis we can, without recourse to parametric assumptions, make basic predictions for how mortality rates in each element of this partition will change as the distribution of education shifts over time. In particular, if an education group shifts downward in the SES distribution, then the mortality rate for that group should increase relative to the population at large. For example, the segment of the SES distribution associated with 12 years of education in 1984-89 ranges from percentile 25.8 to percentile 68.4 , 
whereas in 2002-06 it ranges from the percentile 14.0 to percentile 54.2. Therefore this segment shifted down in the SES distribution. Under the null, mortality should have increased in this segment relative to the population average. In this manner, we can construct a set of testable predictions for the null hypothesis that the SES-mortality gradient has not increased over time.

In practice, we implement our testing procedure separately for each gender using six education groups. Our proposed testing procedure thus amounts to a multiple one-sided hypothesis test for the mortality rates associated with the six different levels of education. ${ }^{5}$ While multiple two-sided hypothesis tests are straightforward to implement using standard methods and software packages, multiple one-sided hypothesis tests of the type we are considering here are less straightforward. In Section 2.2 we adapt results from Kodde and Palm (1986) and Wolak (1989) to our context and thereby derive the required test statistics and critical values.

When we implement this test on the 1984-89 and 2002-06 time periods, we fail to reject the hypothesis that the SES-gradient in mortality has remained unchanged for males, but we do marginally reject the hypothesis of no change in the gradient for females. The failure to find evidence for a change in the SES-mortality gradient for males, however, could reflect a lack of power of our proposed test. We address this lack of power by considering another implication of our null model, which is that any combination of education groups that shifts upward in the rank distribution should have lower mortality. Nevertheless, we still fail to reject the null hypothesis that the gradient has not changed among males using this alternative test. However we strongly reject the null that the SES-mortality gradient among females remained unchanged between 1984-89 and 2002-06.

As a final substantive point, we wish to comment on which part of the support of the SES distribution seems to be responsible for the strong rejection of the null hypothesis among females between 1984-89 and 2002-06. Visually examining the mortality function over the education distribution, we find that the strongest indication against a stable SES-mortality gradient comes from the very bottom of the distribution. Mortality differences between those with a high school diploma only and those with greater educational attainment have remained relatively stable (in absolute numbers) since the 1980s, although there is some indication of an increasing difference between those with a high school diploma and those

\footnotetext{
${ }^{5}$ We implement the test on three rather than just the two time periods discussed in the introduction. Appendix Table A1 presents the information analogous to Table 1 for the full set of education intervals, both genders, and all three time periods.
} 
with a four-year college degree. But it is primarily at the bottom of the education distribution that we observe a widening in the relationship between mortality and education over time.

The remainder of the paper is structured as follows. Section 2 outlines an analytical framework and develops testing procedures for the relationship between SES and mortality. Section 3 describes the data used in this paper. Section 4 presents and describes the results of the statistical tests. Section 5 concludes.

\section{Analytical Framework}

In this section we first model the latent relationship between an individual's socioeconomic status (SES) and her mortality rate. We then use this framework to develop nonparametric testing procedures to examine whether the relationship between mortality and SES has changed over time.

\subsection{Mortality Model}

The literature commonly uses education as the primary marker of SES (e.g., Preston and Taubman, 1994; Hummer and Lariscy, 2011; Olshansky et al., 2012), and we follow this approach. To begin, we define SES directly as an individual's relative position within society. ${ }^{6}$ Accordingly each individual, $i$, is associated with a rank, $r_{i} \in[0,1]$, which expresses her SES. ${ }^{7}$ The exact rank of an individual is unobserved, but we do observe their level of educational attainment, $e_{i}$. Because we take education to be the primary marker of SES, we suppose there is a monotonic relationship between education and SES. ${ }^{8}$ We state this formally with the following assumption.

Assumption 1 The relationship between the discrete levels of educational attainment and the continuous values of latent socioeconomic status is monotonic: $r_{i}<r_{j} \Longrightarrow e_{i} \leq e_{j}$ and $e_{i}<e_{j} \Longrightarrow r_{i}<r_{j}$.

\footnotetext{
${ }^{6} \mathrm{~A}$ thorough discussion of the concept of SES is well beyond our scope. However some notion of relative position appears in most definitions. See Oakes and Rossi (2003) for an interesting review and discussion of issues in defining and measuring SES in research on health outcomes.

${ }^{7}$ Naturally SES may depend on multiple attributes. The existence of a well defined ranking assumes these attributes can be collapsed into a single index.

${ }^{8}$ Which way the causation runs between SES and education is irrelevant for our purposes. We only require that education is an accurate marker of SES in the sense that those individuals with higher education are understood to be of higher SES. Of course if there are multiple attributes behind SES then it need not be monotonic in education. We make this simplifying assumption to facilitate the analysis, and leave for future research the possibility of extending our approach to multiple attributes.
} 
This assumption implies that the observed education distribution partitions the latent SES distribution in the population. Denote by $K$ the number of different levels of educational attainment observed in the data. Based on these categories we can define a partition of the SES distribution.

Definition 1 (Education Partition) An education partition, $P_{t} \in[0,1]^{K+1}$, is a vector at time $t$ with $K+1$ increasing thresholds $p_{t}^{k}$ in the distribution of $S E S$, where $p_{t}^{0} \equiv 0, p_{t}^{K} \equiv 1$, and $k=0, \ldots, K$.

$$
P_{t}=\left(p_{t}^{0}, p_{t}^{1}, \ldots, p_{t}^{K}\right)
$$

For example, the vector associated with the education distribution for females in 1984-89 in Table 1 , column 2 is $P_{1983-88}=(0,0.258,0.684,1){ }^{9}$

We measure mortality as the probability of death over a five-year time period, $t .{ }^{10}$ This is a function of the individual's SES, education, and age, $a_{i t}$, and the function can change over time to reflect both overall changes in mortality and possible changes in the gradient. To define the function, let $D_{i t}$ be an indicator for whether individual $i$ dies during period $t$; then the mortality function can be expressed as follows.

Definition 2 (Mortality Function) A mortality function $m_{t}(\cdot)$ gives the probability of death during period $t$ conditional on SES ( $r)$, educational attainment (e), and age (a).

$$
m_{t}(r, e, a) \equiv \operatorname{Pr}(D=1 \mid r, e, a, t)
$$

Assumption (1) implies that SES fully determines education. Accordingly there is no variation in education conditional on SES that might be used to separately identify the effects of education and SES. Therefore, to proceed with our analysis, we subsume any independent effect of education on mortality in the effect of SES on mortality and write the mortality function as $m_{t}(r, e, a)=m_{t}(r, a) .{ }^{11}$ Additionally, throughout the empirical

\footnotetext{
${ }^{9}$ For this vector, $K=3$. The correspondent vector associated with the education distribution for females with $K=6$, in Appendix Table A1, is $P_{1983-88}=(0,0.122,0.257,0.683,0.819,0.942,1)$.

${ }^{10}$ All subsequent references to an individual's "mortality rate" or their "probability of death" refer to the probability of death within a 5-year time period.

${ }^{11}$ This is clearly not an innocuous assumption. For instance, it is possible that education has a direct causal effect on mortality by enabling individuals to comprehend medical advice or by leading them to make better health decisions (Kenkel (1991); Lange (2011)). The strength of such a link between education and mortality might vary over time. By assuming education to have no independent effect, we focus attention on the relative aspects of individuals in society, not their overall education. If the functional role of education in producing health changes over time, then this will be interpreted by us as a change in the SES gradient. Education is of course a primary determinant and marker of SES and therefore changes in the functional role of education do indeed reflect changes in the SES gradient. In this sense, our interpretation is correct.
} 
analysis we fully adjust for age. Thus, for the remainder of the exposition, we will also keep age implicit and write $m_{t}(r)$.

Assumption (2) below restricts the mortality function to decrease in SES.

Assumption 2 Mortality weakly decreases in SES: $r_{i}>r_{j} \Longrightarrow m\left(r_{i}\right) \leq m\left(r_{j}\right)$.

Together with Assumption (1), this implies that mortality declines with education. The evidence presented in section 3.2 provides strong support for this prediction.

We are interested in discovering how $m_{t}(r)$ changes over time. The problem in answering this question is that we cannot observe an individual's rank, $r$, so we cannot estimate the function $m_{t}(r)$ directly. However, we can calculate the mortality rate for each interval defined by the education partition. In our framework this is expressed as follows:

$$
M_{t}^{k}=\frac{\int_{p_{t}^{k-1}}^{p_{t}^{k}} m_{t}(r) \mathrm{d} r}{p_{t}^{k}-p_{t}^{k-1}}, k=1, \ldots, K
$$

The mortality rate conditional on education approximates the underlying mortality function, $m_{t}(r)$, as a step function. This is illustrated in Figure $1 .{ }^{12}$ For example, consider the middle group in Figure 1. For that group, we know that $r$ lies between 0.3 and 0.7 , and we observe an average five-year mortality rate of 6 percent. The unobserved relation between mortality and $r$ over this segment ranges from approximately 5 to 7 percent.

\subsection{A Nonparametric Test for Changes in the SES-Mortality Gra- dient}

Based on the model above, we develop and implement nonparametric tests of the hypothesis that the gradient of the relationship between SES and mortality, i.e., $m_{t}(r)$, has remained unchanged between two time periods $t_{1}$ and $t_{2}$. The alternative hypothesis is that the gradient has increased over time in some arbitrary fashion.

While we test the null that the SES-mortality gradient is constant, we do allow for a common shift of mortality over time. We could accommodate such a shift using either an additive or multiplicative time-varying term, $g_{t}$, in the mortality function. Under the null, the remainder of the mortality function is constant over time. Hence we formulate the null

\footnotetext{
${ }^{12}$ Figure 1 is not based on real data and is for illustrative purpose only.
} 


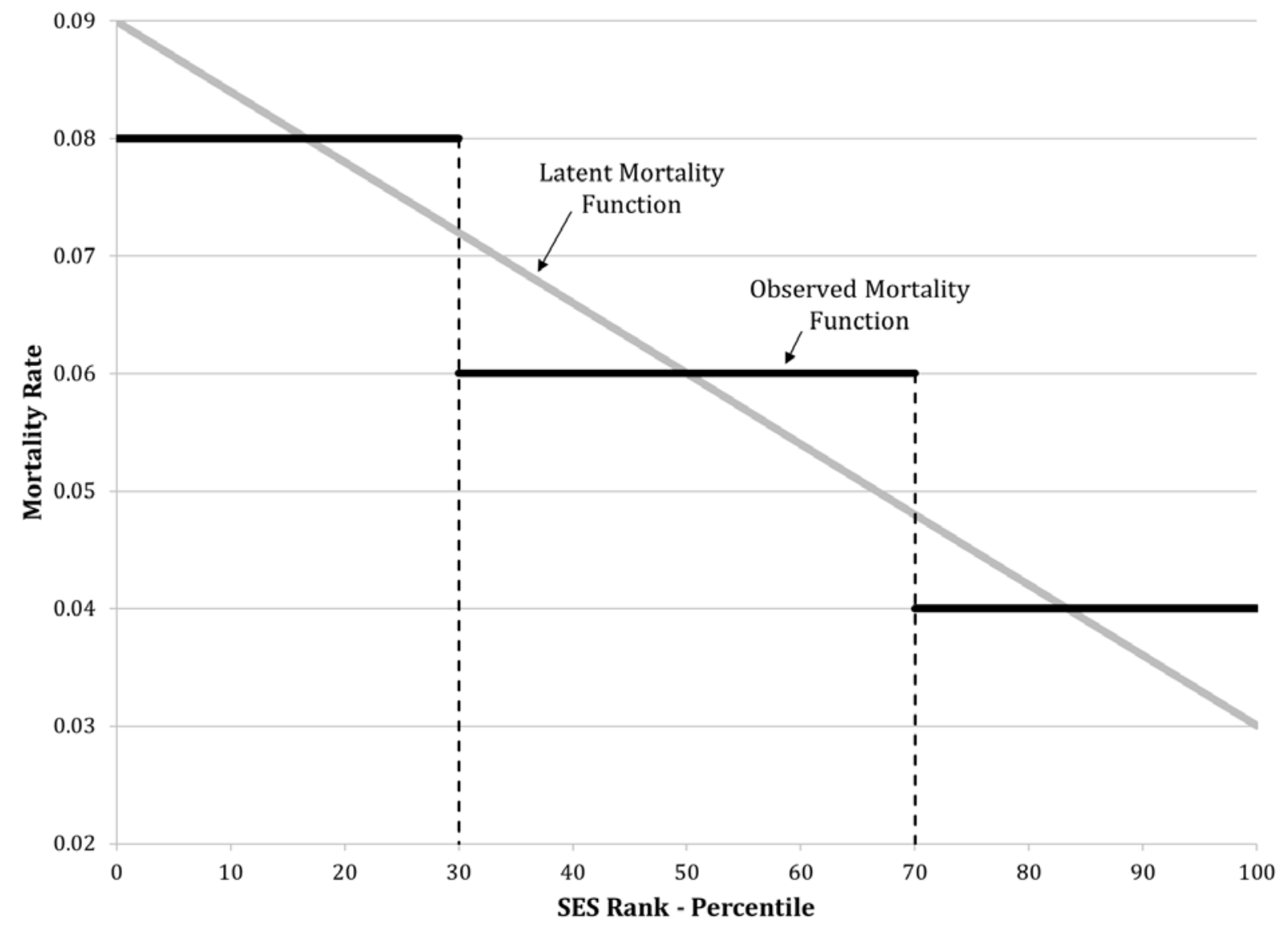

Figure 1: An example of a latent mortality function and an observed mortality function

hypothesis as either:

$$
H_{0}: m_{t}(r)=\bar{m}(r)+g_{t} \quad \text { or } \quad H_{0}: m_{t}(r)=\bar{m}(r) \cdot g_{t} .
$$

In what follows, we focus on the additive case. ${ }^{13}$ The null hypothesis implies that the mortality function at times $t_{1}$ and $t_{2}$ should be identical once we remove a constant term from each period; i.e.,

$$
m_{t_{1}}(r)-g_{t_{1}}=m_{t_{2}}(r)-g_{t_{2}}=\bar{m}(r) .
$$

Therefore we proceed by using mortality functions that are demeaned within each time period. $^{14}$

\footnotetext{
${ }^{13}$ It is simple to extend our methodology to the multiplicative case by taking the log of the mortality rates.

${ }^{14}$ To see how demeaning within each time period yields mortality functions that will be the same across time periods, note that the overall average mortality rate in period $t$ is $\int_{0}^{1} m_{t}(r) \mathrm{d} r=g_{t}+\mu$, where $\mu \equiv \int_{0}^{1} \bar{m}(r) \mathrm{d} r$.
} 
Because the mortality rates at exact ranks $r$ are unobservable, we must work with the average mortality rates for each interval defined by the education partition. To derive a testable implication from the model, we first identify those elements of the partition where the intervals in the distribution of SES shift downward from one time period to the next. These are intervals $\left(p_{t_{1}}^{k-1}, p_{t_{1}}^{k}\right)$ and $\left(p_{t_{2}}^{k-1}, p_{t_{2}}^{k}\right)$ such that $p_{t_{2}}^{k-1}<p_{t_{1}}^{k-1}$ and $p_{t_{2}}^{k}<p_{t_{1}}^{k}$. Then from the monotonicity of $\bar{m}(r)$ we have the implication that

$$
\left(p_{t_{2}}^{k}-p_{t_{2}}^{k-1}\right)^{-1} \int_{p_{t_{2}}^{k-1}}^{p_{t_{2}}^{k}} \bar{m}(r) \mathrm{d} r \geq\left(p_{t_{1}}^{k}-p_{t_{1}}^{k-1}\right)^{-1} \int_{p_{t_{1}}^{k-1}}^{p_{t_{1}}^{k}} \bar{m}(r) \mathrm{d} r
$$

In words, an interval that shifts downward over time would have increasing mortality (in the demeaned mortality function). For example, one can see in Figure 1 that if the intervals were to shift left the mortality rate would increase within each interval. The exact magnitude of this increase is unknown without knowledge of the underlying mortality function; we only know that the average mortality rate must increase. Thus the model yields a one-sided null hypothesis of weakly increasing mortality for each interval that shifts downward over time.

Because this downward shift occurs for multiple education groups, the model yields a joint one-sided prediction under the null hypothesis. The alternative is that mortality decreases for one or more intervals in the partition (relative to the overall mortality rate in each period), even though that interval shifts downward in the distribution of educational attainment. In the data, we observe that all $K$ groups in the education partition shift downward over time. Thus the null hypothesis states that the vector of changes in the demeaned mortality rates for the $K$ education groups lies in the positive orthant of $\mathbb{R}^{K}$. This null is tested against the alternative hypothesis that the vector of changes lies somewhere in the remainder of the space (i.e., some of the changes are negative). Tests for such hypotheses are developed in Kodde and Palm (1986) and Wolak (1989) and are closely related to multiple one-sided tests with point-valued nulls (e.g., Kudo (1963); Gouriéroux et al. (1982)). The Wald statistic for these tests uses only those elements of the vector that violate the null, and these are standardized by the inverse of their variance matrix as in a typical Wald statistic for a two-sided test. The null distribution of this test statistic is a mixture of chi-squares, one for each possible number of violations, where the mixture weights are the probability of that number of violations. As suggested in Wolak (1989), we compute these weights via simulation using the estimated variance matrix for the vector of mortality changes among all groups. (Additional detail is Subtracting this from $m_{t}(r)$ yields $\bar{m}(r)-\mu$, and we can set $\mu=0$ without loss of generality. 
provided in the appendix.)

To compute the test statistic we need the mortality rate (after demeaning mortality across the whole population within each period) for each partition element $k$ in time periods $t=1$ and 2. These mortality rates are standardized to the joint population distribution of age, race, and region in 2000, as noted in Section 3. We recover the demeaned mortality rates and their joint variance matrix using a dummy variable regression that includes a constant term for each time period, $\mu_{t}$, and constrains the coefficients on the indicators for the $K$ education groups to add to zero:

$$
D_{i t}=\mu_{t}+\sum_{k=1}^{K} \beta_{t}^{k} 1\left(k_{i}=k\right)+u_{i t}, t=1,2
$$

where in each period $t$ the sum of the $\beta_{t}^{k}$ weighted by the share of individuals in group $k$ is constrained to be equal to zero (i.e., $\sum_{k} \beta_{t}^{k}\left(p_{t}^{k}-p_{t}^{k-1}\right)=0, t=1,2$ ), and $u_{i t}$ is a mean-zero error term. ${ }^{15}$ The variance matrix estimate is robust to heteroskedasticity and clustered on the individual in cases where an individual appears in multiple time periods.

\subsection{Augmented Test with Upward Shifts}

It is possible that our test has weak power against certain violations of the null hypothesis, particularly from changes in the gradient toward the bottom of the education distribution. Therefore we consider an alternative version of the test that has greater power to detect such changes in the gradient.

To explain this test, note that in its simplest form, an increase in the gradient would be represented as a rotation of a linear relationship, as in Figure 2. Here it is apparent that the power to detect such a change with the above test comes from the top of the education distribution, which is to the right of the intersection of the two underlying mortality functions (the solid gray line in the first period and the dashed gray line in the second period). Only to the right of the intersection is it possible that the mortality rate in an interval might have declined even though the interval shifted downward (i.e., the black dashed line that runs from the 60th to 100th percentile). Thus, violations of the null could only be detected toward the top of the education distribution. We could increase the power of our test against alternatives such as that represented in Figure 2 by reconfiguring the education partition in an appropriate fashion. In particular, we could combine intervals in the later years so

\footnotetext{
${ }^{15}$ Note that $u_{i t}$ has only two points of support because $D_{i t}=0$ or 1 ; i.e., this is a linear probability model.
} 


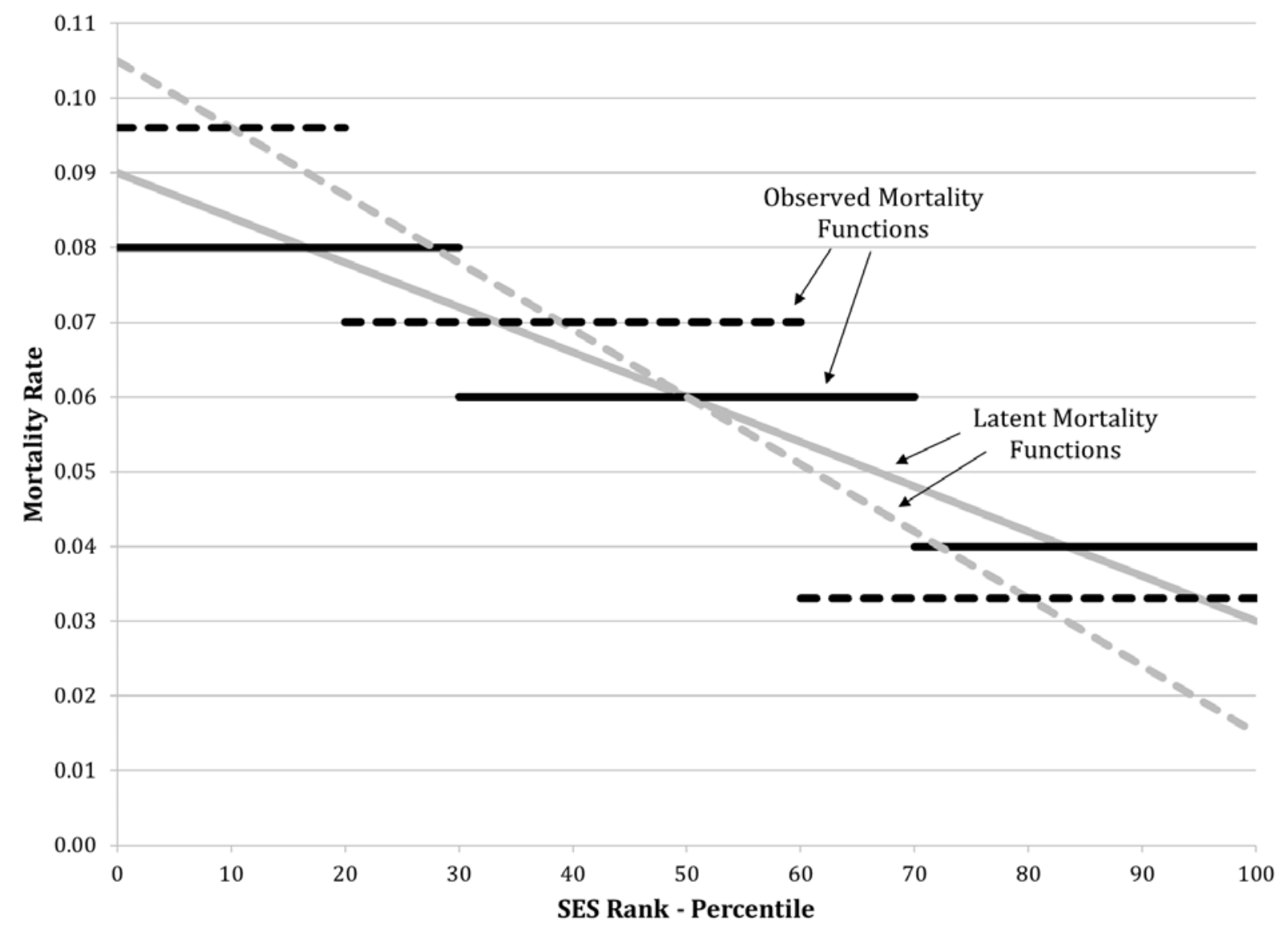

Figure 2: Simple example of a gradient that steepens over time

that we obtain upward shifts in intervals toward the bottom of the education distribution. Under the null, any segment that shifts upward should have lower mortality. Thus for this alternative test we create an interval that shifts upward over time by combining the lowest groups in the education partition in the later time period. Then the full prediction under the null is that mortality should increase for those intervals that shift downward but should decrease for those that shift upward over time. Figure 2 indicates how we could find evidence against this, if for example the lowest interval in the second time period extended to the 30th or 40th percentile, in which case the mortality rate in that combined group would increase.

When we implement this test in our data, we compare the lowest interval in the first period with the combination of the lowest two intervals in the second period. For example, we combine the bottom two intervals in 2002-06 in Figure 3 below, which then extends beyond the lowest interval in 1992-96 and (barely) beyond the lowest in 1984-89. We use 
only this single combination of the first and second elements because the third element, Grade 12 , is a large group that reaches the middle of the distribution, while the power to detect violations in this manner is likely to come from the bottom of the education distribution. We also include the top interval in this test, which shifts downward and therefore is predicted to have increasing mortality. ${ }^{16}$

\section{Data}

\subsection{Sample Description}

Our data come from two sources: the National Longitudinal Mortality Study (NLMS) Public Use Microdata Sample (PUMS) and the National Health Interview Survey (NHIS) linked to the National Death Index (NDI). ${ }^{17}$ The NLMS consists of data from Current Population Surveys in the early 1980s and a subset of the 1980 Census combined with death certificate information. The NHIS sample covers respondents to the NHIS from 1986 to 2000 which have been linked to deaths recorded by the NDI through 2006. ${ }^{18}$ The NLMS and NHIS are particularly useful since educational attainment is self-reported during the year the individual is interviewed in the base sample. This removes the need to rely on education as recorded on death certificates. Education data from death certificates is often missing and known to be subject to nonrandom measurement error (Sorlie and Johnson, 1996). Furthermore, data in the NHIS is gathered during face-to-face interviews, which reduces (but does not eliminate) exaggerated claims of educational attainment (Black et al., 2003).

We restrict our analysis to non-Hispanic blacks and whites between 25 and 84 years old. ${ }^{19}$ Following Cutler et al. (2011), we restrict the analysis to individuals surviving at least one year from the baseline interview. This is necessary since the base samples do not include the institutionalized population, which includes those in nursing homes. The concern is that

\footnotetext{
${ }^{16}$ We only use the top interval, not the top two, because it is unlikely that the intersection between the two underlying mortality functions in some alternative hypothesis would be this high in the SES distribution. Accordingly we may have better power against such alternatives by only using the top interval.

${ }^{17}$ Information on the NLMS is available at: https://www. census.gov/did/www/nlms/index.html. Information on the NDI is available at: http://www.cdc.gov/nchs/ndi.htm. Information on the NHIS linked mortality files is available at: http://www.cdc.gov/nchs/data_access/data_linkage/mortality/nhis_ linkage.htm.

${ }^{18}$ Linked mortality data to NHIS surveys are available from the date of NHIS interview through the last quarter (October-December) of 2006.

${ }^{19}$ Age was top-coded at 85 years in the NHIS from 1997 onward, so we restrict to a maximum of 84 years of age in order to include individuals from the more recent survey years.
} 
Table 2: Construction of mortality time periods by data source

\begin{tabular}{lllc}
\hline Data Source & Survey Years & Mortality Time Period & Sample Size \\
\hline NLMS & Early 1980s & April 1, 1984- March 31, 1989 & 674,539 \\
NHIS & $1986-1990$ & January 1, 1992-December 31, 1996 & 280,666 \\
NHIS & $1986-2000$ & January 1, 2002-December 31, 2006 & 686,645 \\
\hline
\end{tabular}

the institutionalized have higher death rates than the rest of the population. Conditional on surviving one year, mortality rates for the institutionalized population closely resemble those for the entire population (Meara et al., 2008). All mortality rates are standardized to the age, race, and Census region distribution in the NHIS survey in 2000 and are calculated separately by gender, so that trends do not reflect changes in the distribution of these characteristics. ${ }^{20}$

We use our two data sources to estimate mortality rates in three 5-year time periods, which allow us to analyze trends in mortality by SES rank over time. Our construction of the three mortality time periods is summarized in Table 2. All records in the NLMS are assigned a common starting point of April 1, 1984. We calculate mortality rates by identifying those in the sample who died during the subsequent 5-year period. The precise mortality time period is April 1, 1984 to March 31, 1989, which we abbreviate as 1984-89. We construct two 5-year mortality time periods from the NHIS, from January 1, 1992 to December 31, 1996 and from January 1, 2002 to December 31, 2006. For the former we pool the annual interview samples from 1986 to 1990, and for the latter we use all available interview samples from 1986 to 2000. Our three constructed samples all consist of individuals who are alive at the start of their respective mortality time period.

Prior to 1992, the CPS asked for the highest grade level a respondent had attended, and a separate question verified whether the respondent had actually completed that grade. In the NLMS, grade levels are grouped at lower levels; categories include grade 1 through grade 4 , grade 5 and grade 6 , and grade 7 and grade 8 . Grades 9 through 6 years of post-secondary education are available year-by-year.

From 1986 to 1996, educational attainment in the NHIS was measured as the highest completed year of schooling ranging from 1 year of schooling to 18 or more years of schooling. From 1997 to 2000, the concept used to measure educational attainment changed to an educational achievement approach, using a combination of years of schooling as well as

\footnotetext{
${ }^{20}$ Montez and Berkman (2014) find subtle differences by region in trends in the education-mortality gradient.
} 
specific degrees attained such as "some college but no degree," "associate degree," and "bachelor's degree." The highest completed year of schooling ranges from 1 year of schooling to 12 years of schooling, followed by a range of post-secondary options. We recoded the postsecondary options as follows: some college but no degree $=13$ years of schooling; associate degree $=14$ years of schooling; bachelor's degree $=16$ years of schooling; master's degree, professional degree, or doctoral degree $=18$ years or more of schooling.

For the main analysis we divide the population into six groups by educational attainment. The definitions of four of the six education groups do not change across the three constructed

samples; they are: individuals who completed grade 12, those who completed some college (1 to 3 years, with or without an associate degree), those who completed 4 years of college, and those with more than 4 years of post-secondary education. The definitions of the remaining two groups at the bottom of the education distribution change slightly across the three constructed samples in order to keep the fraction in the lowest group approximately constant over time. For the 1984-89 mortality time period, these two groups are individuals who completed grade 8 and below, and those who completed grade 9 through grade 11 . For 1992-96, they are those who completed grade 9 and below, and those who completed grade 10 and grade 11 . For 2002-06, the two groups are those who completed grade 10 and below, and those who completed grade 11.

\subsection{Education and Mortality Rates: The Raw Data}

Appendix Table A1 displays the 5-year standardized mortality rates (by age, race, and region) and the population shares in each education group and in each time period. The same information is displayed graphically in Figure 3 for males and Figure 4 for females. In the discussion that follows we refer to the statistics given in the appendix table and displayed in these two figures.

Figure 3 shows male mortality by percentiles of educational attainment for the three samples from different time periods defined in Table 2. There are six education groups that partition the distribution of educational attainment. The share of the population in each education group corresponds to the length of the horizontal line segments. For instance, we see that the share of males with more than 4 years of post-secondary education increased slightly from $10.4 \%$ in $1984-89$ to $11.4 \%$ in $1992-96$ and finally $12.0 \%$ in $2002-06$. The average mortality rate for each group is represented on the vertical axis. For example, we see that the average mortality of males with more than four years of post-secondary education 


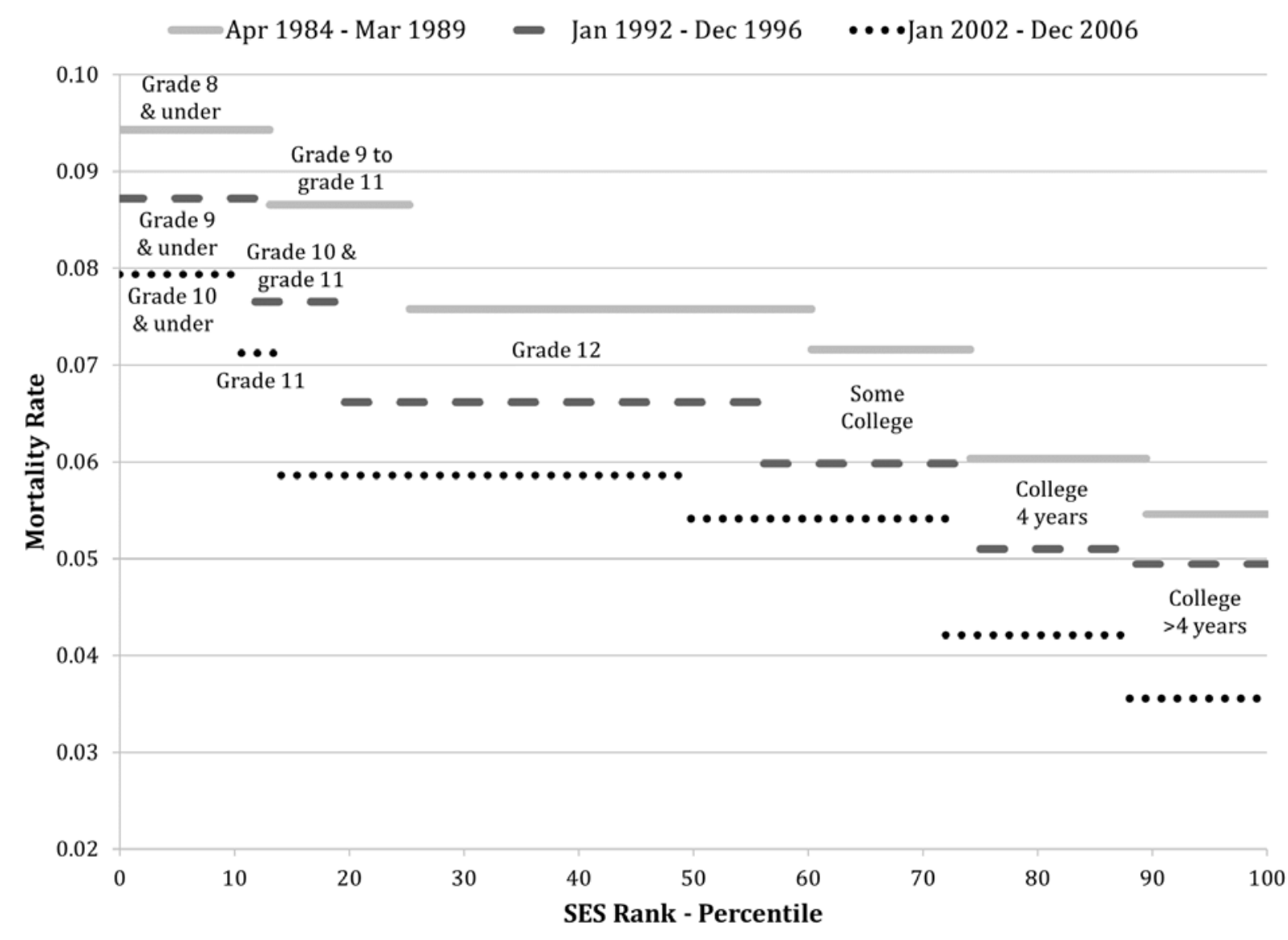

Figure 3: Observed mortality rates by SES for males (1984-2006)

fell relatively slowly from 0.055 to 0.049 between the 1980 s and $1990 \mathrm{~s}$ and then decreased more rapidly to 0.036 between the 1990s and 2000s.

Figure 3 illustrates three findings regarding the relation between mortality and education over time. First, mortality declined rapidly over the last three decades across the education distribution. Second, there are large disparities in mortality by education and these disparities persist over time. A third finding apparent from Figure 3 is that mortality in both absolute and relative terms declined more for males at the top of the education distribution. Between 1984-89 and 2002-06, mortality rates among those with more than four years of college decreased by 1.8 percentage points from a base of 5.3. Furthermore, they decreased by 1.6 percentage points among those with exactly four years of college. By contrast, over the same time period the mortality rates among those with the lowest and second lowest level of educational attainment declined by only 1.1 and 1.2 percentage points respectively. 


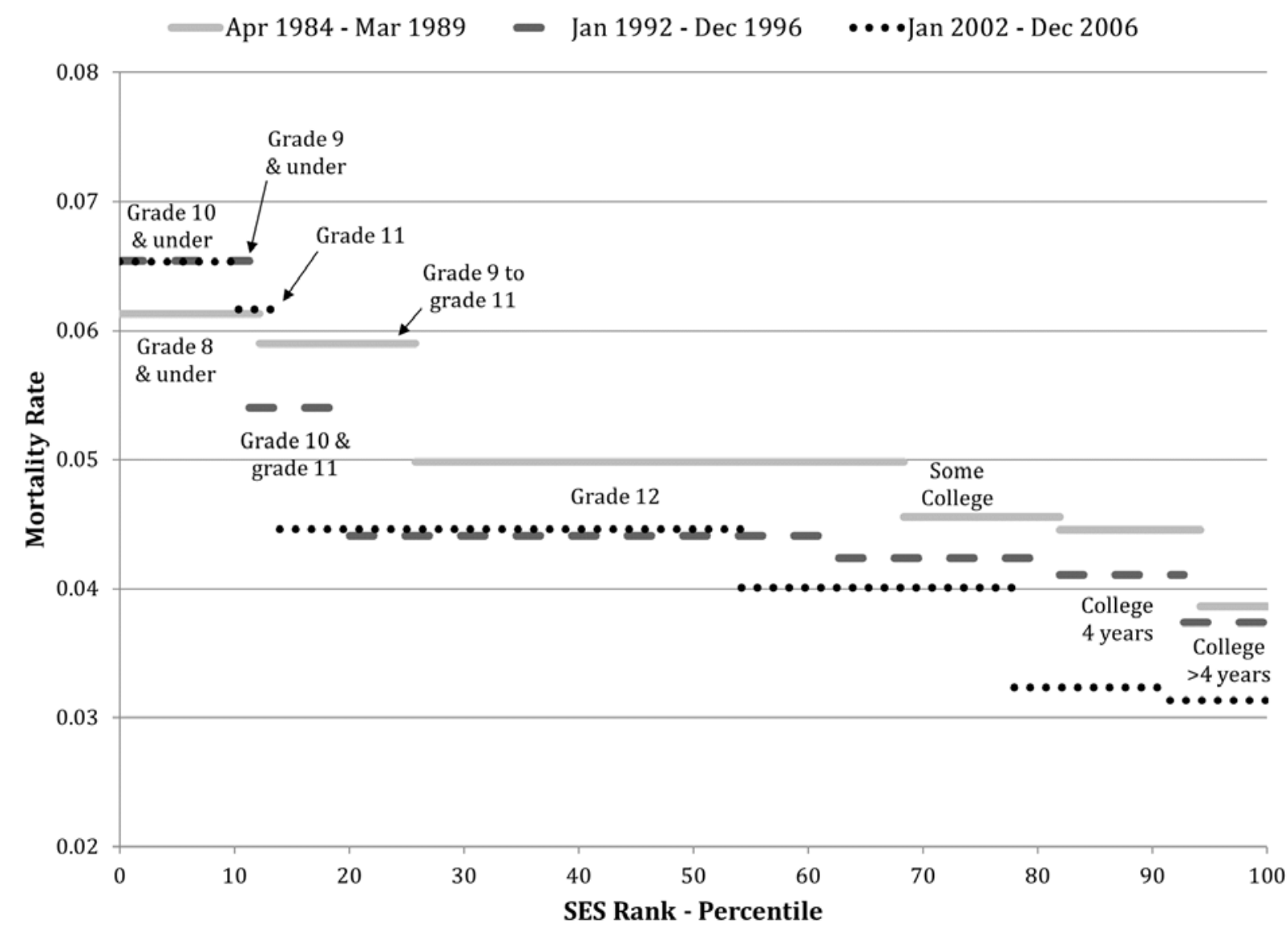

Figure 4: Observed mortality rates by SES for females (1984-2006)

Figure 4 mirrors Figure 3 but for females. As for males, we find that there are large disparities in mortality by education in all three periods. However, there are several striking differences between females and males. First, while average female mortality by educational attainment is substantially lower than for males, female mortality has declined less between the 1980s and 2000s. Second, the declines in female mortality were much more concentrated among women at the top of the education distribution. Indeed, mortality rates among the least educated women seem to have increased between 1984-89 and 2002-06. By contrast, among males mortality rates declined at all levels of educational attainment. We cannot be sure, of course, whether the increase in the mortality rates among the least educated women simply reflects the shift down in SES associated with these education groups. This is the question we address next with the results from our testing procedure. 


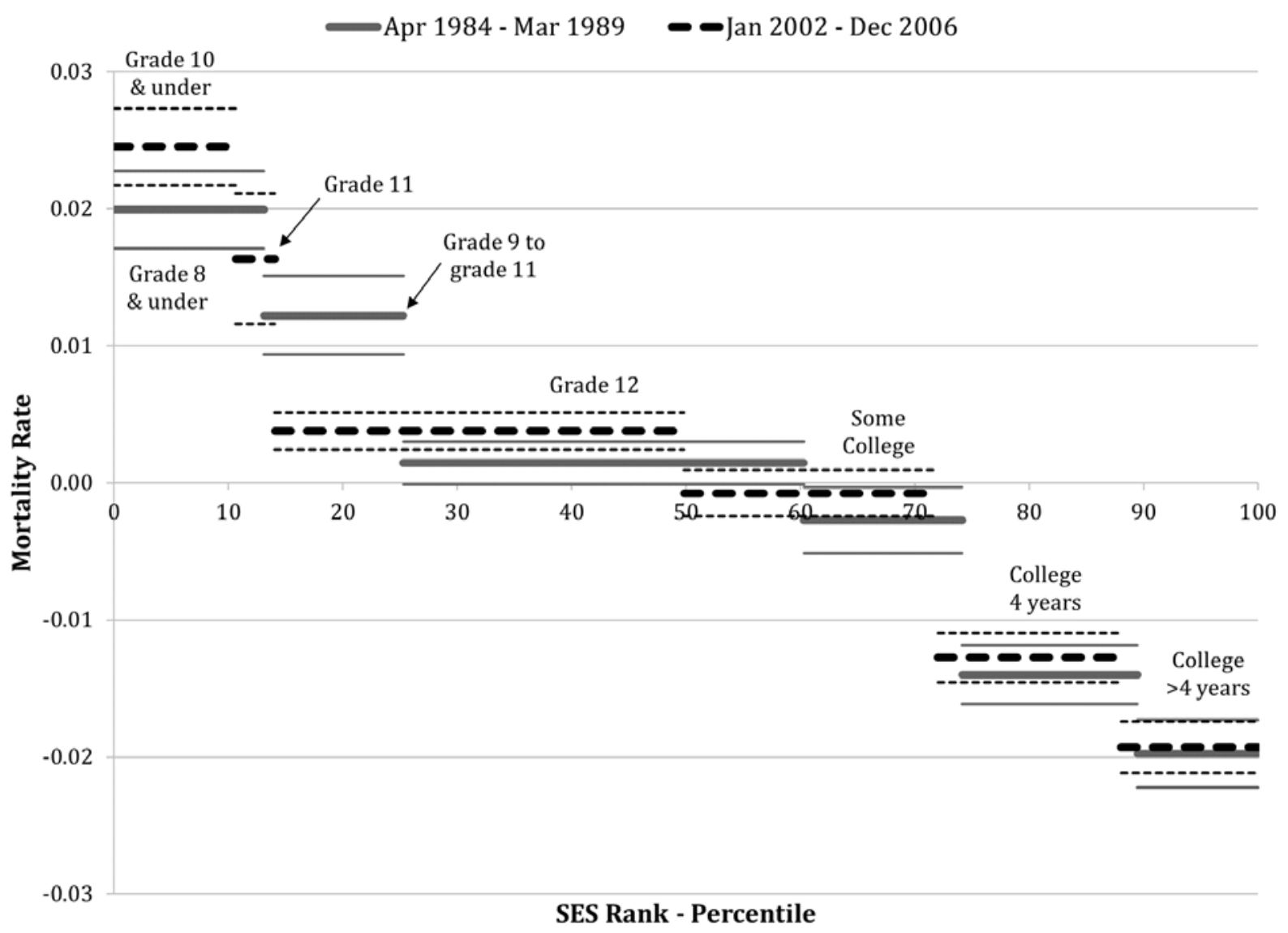

Figure 5: Demeaned mortality rates by SES for males (1984-2006)

\section{Main Results}

In Figures 5 and 6 we show male and female mortality functions for 1984-89 and 2002-06 with $95 \%$ confidence intervals. The mortality functions in both periods have been demeaned and therefore average to zero within each period. These figures show the mortality functions we use to implement our testing procedures.

We can compare the mortality functions between the two time periods in each figure to see which segments of the education partition violate the null. Such a violation occurs if a segment that shifts downwards (to the left) in the education distribution has lower mortality in 2002-06 than in 1984-89. For males none of the education groups violates the prediction under the null; instead all segments show higher (demeaned) mortality in 200206. Thus we find no evidence for an increase in the SES-mortality gradient among males 


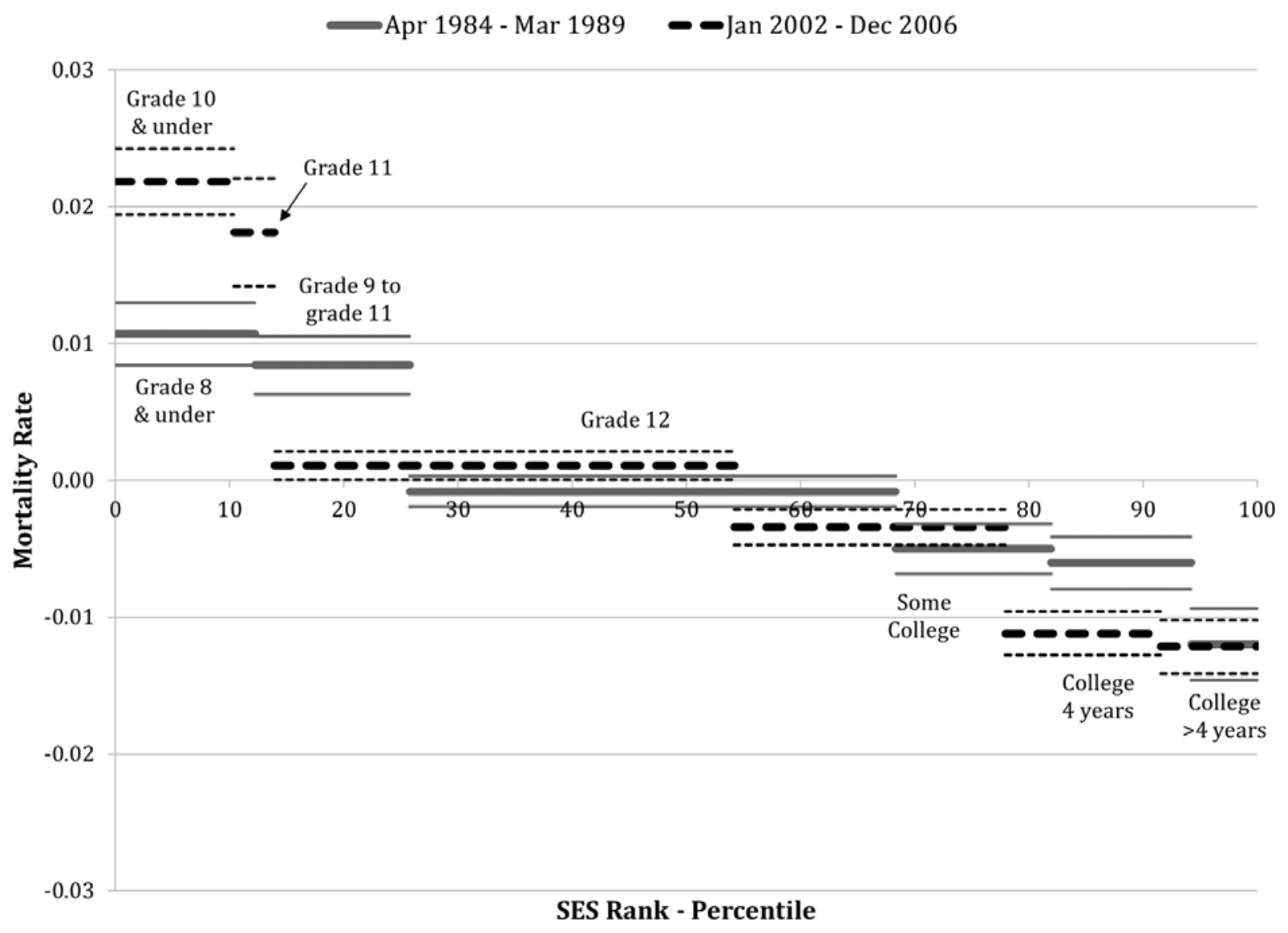

Figure 6: Demeaned mortality rates by SES for females (1984-2006)

using our first testing procedure. Correspondingly, Table 3 reports that the test statistic for males between 1984-89 and 2002-06 is 0. Comparing the 1990s with the 2000s does yield a violation of the null, but the test statistic for this comparison is much smaller than the critical value. For females, we observe in Figure 6 that mortality declined among the top two education groups from 1984-89 to 2002-06 despite these segments shifting downward in the education distribution. This decline in relative mortality was fairly substantial for females with exactly four years of education. Nevertheless, the test statistic for the basic test applied to this time span is marginally below its critical value. On the other hand, the statistic from the test comparing 1992-96 to 2002-06 is marginally above its critical value, thereby formally rejecting the hypothesis of no change in the gradient for females over that time span. The top panel in Table 3 thus reveals weak evidence that the gradient increased among women and no evidence that it increased among men. 
Table 3: One-sided statistical test results

\begin{tabular}{|c|c|c|c|c|c|c|}
\hline & \multicolumn{3}{|c|}{ Female } & \multicolumn{3}{|c|}{ Male } \\
\hline & $1980 \mathrm{~s}-1990 \mathrm{~s}$ & $1990 s-2000 s$ & $1980 s-2000 s$ & $1980 \mathrm{~s}-1990 \mathrm{~s}$ & $1990 \mathrm{~s}-2000 \mathrm{~s}$ & $1980 s-2000 s$ \\
\hline \multicolumn{7}{|c|}{ Basic test using only decreasing education segments } \\
\hline Statistic & 2.23 & 8.50 & 8.00 & $0^{*}$ & 3.06 & $0^{*}$ \\
\hline$\{5 \%$ c.v $\}$ & $\{8.28\}$ & $\{8.24\}$ & $\{8.21\}$ & $\{6.34\}$ & $\{8.21\}$ & $\{8.19\}$ \\
\hline$[p$-value $]$ & {$[0.513]$} & {$[0.045]$} & {$[0.055]$} & {$[0.976]$} & {$[0.383]$} & {$[>0.999]$} \\
\hline \multicolumn{7}{|c|}{ Augmented test using a combination of increasing and decreasing segments } \\
\hline Statistic & 3.76 & 1.67 & 39.16 & $0^{*}$ & 3.06 & 1.56 \\
\hline$\{5 \%$ c.v $\}$ & $\{4.28\}$ & $\{4.27\}$ & $\{4.27\}$ & $\{4.28\}$ & $\{4.27\}$ & $\{4.27\}$ \\
\hline$[p$-value $]$ & {$[0.066]$} & [0.208] & {$[<0.001]$} & {$[0.718]$} & [0.096] & {$[0.221]$} \\
\hline
\end{tabular}

Notes: c.v. is the critical value. The precise start and end dates of each time period are given in Table 2.

* There were no violations of the null hypothesis in these comparisons, hence the value of the test statistic is zero by definition. However, the p-values are less than one because there is positive mass at zero in the distribution of the test statistic under the null.

Figure 6 illustrates why our first test could be underpowered in detecting changes in the mortality gradient. The mortality rates in the two lowest groups, grade 10 and under and grade 11, are much higher relative to the population average in 2002-06 than the mortality rate in the lowest group, grade 8 and under, in 1984-89. However, since these segments shifted to the left, this substantial increase in relative mortality among the least educated can be explained as resulting either from a change in the SES gradient or from a change in selectivity associated with education. Therefore it does not contribute to the test statistic and will not be detected by our first test. Since the first test exclusively relies on segments that shift left and for whom relative mortality declined, it is not well designed to detect changes in the gradient that manifest themselves as relative increases in mortality at the bottom of the distribution. It is this lack of power that the augmented test described in Section 2.3 is designed to address. The augmented test combines segments at the bottom of the partition to obtain additional testable implications from upward shifts in the education distribution. Using the augmented test, we strongly reject the absence of a widening of the SES-mortality gradient among females from 1984-89 to 2002-06 (see Table 3). Among males we still fail to reject the null of no change in the gradient, both for the full time span and each shorter time span. Thus our results provide strong evidence that the SES-mortality gradient 
widened among females over the last thirty years, even once changes in the distribution of educational attainment are accounted for. By contrast, among males any evidence that the SES-mortality gradient increased over this time is marginal at best.

Our tests do not identify where in the SES distribution the change in the gradient among females is occurring, but the results above provide clear though informal evidence toward this point. The augmented test derives additional power from exploiting the changes in mortality at the lower end of the distribution of educational attainment. The fact that the strongest evidence that the SES-mortality gradient widened comes from this test, rather than the basic test, thereby suggests that the increase in the gradient among females is driven more by changes at the bottom of the SES distribution than at the top. Visual inspection of Figures 4 and 6 strongly suggests this as well. Thus it appears that the widening of the SES-mortality gradient among females was driven primarily by the smaller gains in mortality among the less educated.

\section{Conclusion}

The evolution of inequality increasingly holds a central place in societal discourse. In recent years this fact was demonstrated by the rise of the "Occupy" movement, which dominated headlines in late 2011, and by the tremendous success of Thomas Piketty's Capital in the Twenty-First Century (Piketty, 2014), which reached number one on the New York Times bestseller list. Within this context, the finding that the mortality difference between the least and the most educated has increased substantially since the 1980s has seemed to strike a nerve. It has generated substantial interest both in academic circles and in the popular press (e.g., Tavernise, 2012). Finding an increasing mortality-education gradient reinforces the perception of an increasingly polarized, unequal society. If mortality inequalities in the population widened substantially, then the possibility arises that not only did the less well-off not benefit from the increase in material wealth over the last few decades, but they might also have failed to benefit from the tremendous progress made over the last half-century in reducing mortality. The question of whether mortality inequality increased in the population is thus of primary importance for understanding broader social trends.

In this paper, we ask whether evidence that the mortality-education gradient has increased can be taken as evidence that mortality inequality associated with underlying socioeconomic status has widened as well. Our contribution is to ask critically whether the evidence based on the mortality-education gradient is informative about the relation between 
mortality and SES once one takes into consideration that the education distribution itself has shifted tremendously over the last few decades.

Our approach to this problem is to impose only a minimal set of assumptions on the relationship between mortality and SES. The sole restriction we impose on the mortality function is that it decreases weakly in SES. Since we rely on a minimal set of assumptions, we can interpret any evidence we find for an increase in the SES-mortality gradient in a strong way. Using our minimal set of assumptions, we derive two testing procedures of the null hypothesis that the SES-gradient in mortality has remained constant over time. We implement these tests using data from 1984-89, 1992-96, and 2002-06.

Our results are mixed. It is difficult to establish firm evidence that the SES-gradient in mortality has increased among males. A simple visual inspection of Figure 3 shows why this is the case: for males, mortality decreased among all education groups in a fairly stable and regular manner. Thus we find little evidence that mortality inequalities indeed increased among males between 1984 and 2006. On the contrary, we find strong evidence that the SES-mortality gradient increased among females using one of our two tests. This evidence stems largely from the lack of progress in reducing mortality rates at the bottom of the SES-distribution, evident in Figure 4. Since 1984, mortality rates among the least educated women increased and this increase is large enough and cuts across enough of the education distribution that it is difficult to explain as simply the result of increased negative selectivity at the bottom of the education distribution.

Substantively, we believe that this paper should help focus future research effort toward the question of why women at the bottom of the education distribution have failed to benefit from the decrease in mortality that took place between 1984 and 2006 among more educated women, and among the male population irrespective of education. In addition our findings suggest that inequality in mortality has not increased entirely in tandem with inequality in income. At least among males, the evidence points to a steady decline in mortality rates across the entire SES distribution. 


\section{References}

Black, D., S. Sanders, and L. Taylor (2003). Measurement of Higher Education in the Census and Current Population Survey. Journal of the American Statistical Association 98, 545554 .

Cutler, D. M., F. Lange, E. Meara, S. Richards-Shubik, and C. J. Ruhm (2011). Rising educational gradients in mortality: The role of behavioral risk factors. Journal of Health Economics 30, 1174-1187.

Dowd, J. B. and A. Hamoudi (2014). Is life expectancy really falling for groups of low socioeconomic status? Lagged selection bias and artefactual trends in mortality. International Journal of Epidemiology 43(4), 983-988.

Gouriéroux, C., A. Holly, and A. Monfort (1982). Likelihood Ratio Test, Wald Test, and Kuhn-Tucker Test in Linear Models with Inequality Constraints on the Regression Parameters. Econometrica 50(1), 63-80.

Hummer, R. A. and J. T. Lariscy (2011). Educational Attainment and Adult Mortality. In R. G. Rogers and E. M. Crimmins (Eds.), International Handbook of Adult Mortality, pp. 241-261. Springer.

Kenkel, D. S. (1991). Health Behavior, Health Knowledge and Schooling. Journal of Political Economy 99(2), 287-305.

Kitagawa, E. M. and P. M. Hauser (1973). Differential Mortality in the United States: A Study in Socioeconomic Epidemiology. Harvard University Press.

Kodde, D. A. and F. C. Palm (1986). Wald Criteria for Jointly Testing Equality and Inequality Restrictions. Econometrica 54(5), 1243-1248.

Kudo, A. (1963). A Multivariate Analogue of the One-Sided Test. Biometrika 50(3/4), 403-418.

Lange, F. (2011). The role of education in complex health decisions: evidence from cancer screening. Journal of Health Economics 30(1), 43-54.

Meara, E. R., S. Richards, and D. M. Cutler (2008). The Gap Gets Bigger: Changes in Mortality And Life Expectancy, By Education, 1981-2000. Health Affairs 27(2), 350-360. 
Montez, J. K. and L. F. Berkman (2014). Trends in the Educational Gradient of Mortality Among US Adults Aged 45 to 84 Years: Bringing Regional Context Into the Explanation. American Journal of Public Health 104(1), e82-e90.

National Center for Health Statistics (May 2009). The National Health Interview Survey (1986-2004) Linked Mortality Files, mortality follow-up through 2006: Matching Methodology. National Center for Health Statistics, Office of Analysis and Epidemiology. Hyattsville, MD.

Oakes, J. M. and P. H. Rossi (2003). The measurement of SES in health research: current practice and steps toward a new approach. Social Science \&3 Medicine 56 (4), 769-784.

Olshansky, S. J. (2013). Life expectancy and education: The author replies. Health Affairs 32(4), 822 .

Olshansky, S. J., T. Antonucci, L. Berkman, R. H. Binstock, A. Boersch-Supan, J. T. Cacioppo, B. A. Carnes, L. L. Carstensen, L. P. Fried, D. P. Goldman, et al. (2012). Differences In Life Expectancy Due To Race And Educational Differences Are Widening, And Many May Not Catch Up. Health Affairs 31(8), 1803-1813.

Piketty, T. (2014). Capital in the Twenty-First Century. Harvard University Press.

Preston, S. H. and P. Taubman (1994). Socioeconomic Differences in Adult Mortality and Health Status. In L. G. Martin and S. H. Preston (Eds.), Demography of Aging, pp. 279-318. National Academy Press.

Sorlie, P. D. and N. J. Johnson (1996). Validity of educational information on the death certificate. Epidemiology 7, 437-439.

Tavernise, S. (September 20, 2012). Life Spans Shrink for Least-Educated Whites in the U.S. New York Times. Retrieved from http://www.nytimes.com.

Wolak, F. A. (1989). Testing Inequality Constraints in Linear Econometric Models. Journal of Econometrics 41(2), 205-235. 


\section{Appendix}

Here we provide further detail on how our test statistic is obtained and how we determine the critical values and $p$-values reported in Table 3. For the main test using only downward shifts in the education partition, we start with the full vector of differences in the demeaned mortality rates:

$$
\left[\left(\hat{\beta}_{t^{\prime}}^{1}-\hat{\beta}_{t}^{1}\right), \ldots,\left(\hat{\beta}_{t^{\prime}}^{K}-\hat{\beta}_{t}^{K}\right)\right]
$$

where $t$ and $t^{\prime}$ are the first and second time periods, the superscripts indicate the partition elements, and $K=6$ for the six education groups used in each period. Because all partition elements shift downward over time (except for males in the comparison from 1984-89 to 1992-96), the null hypothesis is $\left(\hat{\beta}_{t^{\prime}}^{k}-\hat{\beta}_{t}^{k}\right) \geq 0$ for $k=1 \ldots K$. The test statistic is like a typical Wald statistic, which multiplies elements of this vector of differences by the inverse of their variance matrix, but using only those elements that are negative and hence violate the null.

It is straightforward to construct the test statistic using the realized value of the vector $\left[\left(\hat{\beta}_{t^{\prime}}^{1}-\hat{\beta}_{t}^{1}\right), \ldots,\left(\hat{\beta}_{t^{\prime}}^{K}-\hat{\beta}_{t}^{K}\right)\right]$ and its estimated variance-covariance matrix. It is more difficult to obtain the null distribution of this statistic. Following Kodde and Palm (1986) and Wolak (1989), the null distribution of the test statistic is a mixture of chi-squares with zero to $K$ degrees of freedom, which correspond to the number of possible violations of the null hypothesis in a $K$-dimensional vector. To understand this intuitively, note that under the null, it is possible to observe $0,1,2$ or up to $K$ segments for which $\left(\hat{\beta}_{t^{\prime}}^{k}-\hat{\beta}_{t}^{k}\right)<0$ even though the null holds. Thus, under the null, the test statistic is a mixture of $K$ chi-squared random variables with degrees of freedom varying from 0 to $K$. The mixture weight on the $\chi^{2}$ with exactly $n$ degrees of freedom is given by the probability of observing exactly $n$ violations if the true differences $\left(\hat{\beta}_{t^{\prime}}^{k}-\hat{\beta}_{t}^{k}\right)$ are all exactly zero (i.e., on the boundary of the space for the null hypothesis). These probabilities of observing exactly $k=0,1, \ldots, K$ violations are difficult to obtain in closed form and depend on the variance-covariance matrix of the estimated coefficients. We compute these weights via simulation as suggested in Wolak (1989). Accordingly we simulate draws from a $K$-dimensional multivariate normal with mean zero and variance matrix equal to the estimated variance matrix for the vector of differences, $\left[\left(\hat{\beta}_{t^{\prime}}^{1}-\hat{\beta}_{t}^{1}\right), \ldots,\left(\hat{\beta}_{t^{\prime}}^{K}-\hat{\beta}_{t}^{K}\right)\right]$, and then count the number of negative elements in each draw. The proportion of draws with exactly $n$ negative elements is a close approximation to the probability that the test statistic would have the same number of violations under the null, and so these proportions serve as the weights in the mixture of chi-squares. Finally, the 
critical value is determined as the threshold such that the weighted sum of the probability mass above this point in a series of chi-square distributions with 0 to $K$ degrees of freedom equals the desired significance level. The $p$-values analogously give the weighted sum of the probability mass above the observed value of the test statistic.

For the augmented test we use the difference between the demeaned mortality rates in the lowest education group in the first period, $\hat{\beta}_{t}^{1}$, and the lowest two groups in the second period, $\hat{\beta}_{t^{\prime}}^{1,2}$, along with the difference between the rates in the top education group in both periods. Thus the vector of differences has only two elements:

$$
\left[\left(\hat{\beta}_{t^{\prime}}^{1,2}-\hat{\beta}_{t}^{1}\right),\left(\hat{\beta}_{t^{\prime}}^{K}-\hat{\beta}_{t}^{K}\right)\right]
$$

The null hypothesis is $\left(\hat{\beta}_{t}^{1}-\hat{\beta}_{t^{\prime}}^{1,2}\right) \geq 0$ for the first element because it shifts upward (notice that the order of $t$ and $t^{\prime}$ has been reversed) and $\left(\hat{\beta}_{t^{\prime}}^{K}-\hat{\beta}_{t}^{K}\right) \geq 0$ as before for the second element. We compute the mixture weights as before, but now simulating from a bivariate normal with the appropriate variance matrix. 


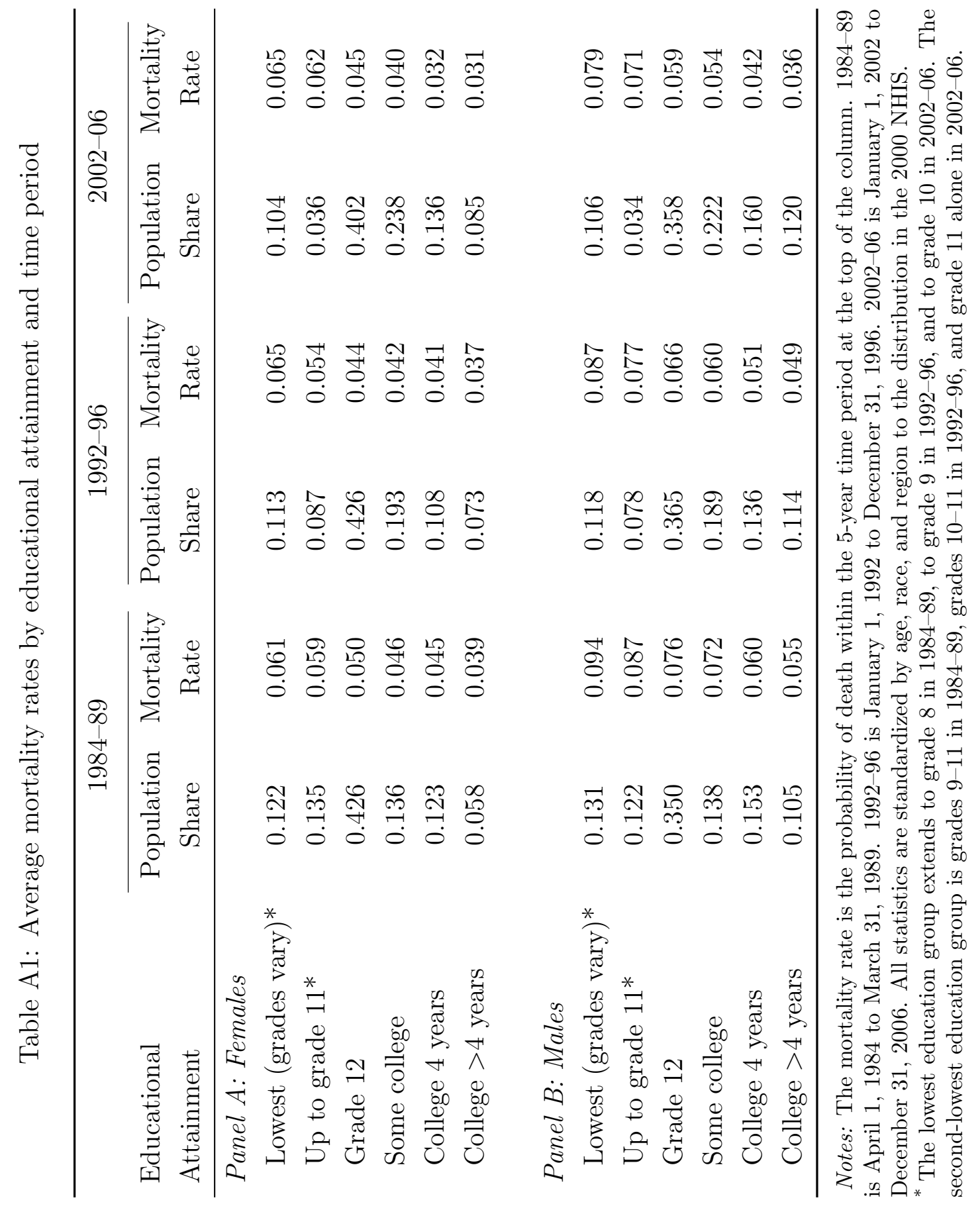

